\title{
Accelerated Junctional Rhythm on a Routine Electrocardiogram: An Unusual Presentation of Cardiac Sarcoidosis
}

\author{
Srikanth Seethala ${ }^{\mathrm{a}}$, Kara Albright ${ }^{\mathrm{b}}$, Srinivasa V. Jampana ${ }^{\mathrm{b}}$ and Jan Němec*,c \\ ${ }^{a}$ Department of Internal Medicine, Texas Tech University Health Sciences Center-El Paso; ${ }^{b}$ University of Pittsburgh- \\ MedicalCenters-MercyHospital; ${ }^{c}$ Cardiovascular Institute, University of Pittsburgh, 200 Lothrop St, Pittsburgh, PA \\ 15213, USA
}

\begin{abstract}
Cardiac sarcoidosis is an uncommon cause of cardiac arrhythmias and cardiomyopathy. We describe a case of cardiac sarcoidosis presenting as an accelerated junctional rhythm and complete atrioventricular (AV) block, without severe left ventricular (LV) dysfunction or pulmonary symptoms. Computer tomography (CT) of the chest and mediastinal lymph node biopsy confirmed the diagnosis. Dual-chamber implantable cardioverter-defibrillator was implanted due to reported risk of sudden cardiac death due to ventricular tachyarrhythmia despite negative programmed ventricular stimulation.
\end{abstract}

Keywords: Cardiac sarcoidosis, Accelerated junctional rhythm, AV block.

\section{CASE REPORT:}

A 66-year old male was noted to have an abnormal electrocardiogram (ECG) during a routine insurance exam. Subsequent ECG obtained in his primary care physician's office revealed junctional rhythm at 56 beats/minute (bpm) and a slightly faster sinus rhythm with atrioventricular (AV) dissociation, suggestive of possible heart block (Fig. 1a). An ECG obtained during another office visit again showed AV dissociation, this time with accelerated junctional rhythm faster than sinus bradycardia, but with a definite blocked $\mathrm{P}$ wave (Fig. 1b). Accelerated junctional rhythm with AV dissociation and possible complete heart block was also present on 24 hour Holter monitoring (heart rate $46-93 \mathrm{bpm}$, average $63 \mathrm{bpm}$ ). Total of 150 premature ventricular complexes were detected over 24 hours, some of the in couplets or in a pattern of trigeminy.

The patient did not have any complaints, although he admitted some decrease in exercise tolerance and "funny feeling" in his chest for 3 months. He was able to exercise in a gym fairly vigorously, though he would recently notice dyspnea of short duration at the onset of physical activity if exercising without a warm-up. He denied any other cardiac or pulmonary symptoms.

This patient was a former smoker and had a history of hypertension and hyperlipidemia. He had a coronary angiogram three years earlier for a pre-syncopal event. That was only significant for $70 \%$ stenosis of the $1^{\text {st }}$ septal perforator. $\mathrm{He}$ had a normal ECG during that admission. Treatment with aspirin and statin was as instituted at that time. An echocardiogram performed a year prior to the current visit showed low normal left ventricular ejection fraction of $49 \%$ with no regional wall motion abnormalities.

*Address correspondence to this author at the Cardiovascular Institute, University of Pittsburgh University of Pittsburgh, 200 Lothrop St, Pittsburgh, PA 15213, USA; Fax: 412-647-7979; E-mail: nemecj@upmc.edu
Placement of dual-chamber pacemaker was considered because of probably symptomatic complete heart block. An echocardiogram repeated prior to pacemaker implantation again showed low-normal left ventricular ejection fraction (LVEF) with regional wall motion abnormalities and mild $\mathrm{LV}$ enlargement ( $\mathrm{LV}$ diastolic diameter of $60 \mathrm{~mm}$ ).

A baseline chest roentgenogram (CXR) performed on the day of the device placement (Fig. 2a, b) was initially read as normal, but its review by the electrophysiologist raised a concern about hilar adenopathy. Computer tomography scan (CT) of the chest without contrast confirmed the presence of hilar adenopathy (Fig. 2d) and also revealed interstitial lung disease (Fig. 2c). A clinical diagnosis of sarcoidosis with cardiac involvement was made.

After a thorough discussion of the new findings with the patient, we decided to proceed with implantation of a dualchamber implantable cardioverter-defibrillator (ICD) instead of dual chamber pacemaker, due to the risk of ventricular tachyarrhythmias in cardiac sarcoidosis. The procedure was completed uneventfully.

Mediastinoscopy with biopsy of anterior tracheal lymph nodes was performed 2 months after ICD placement. Biopsy revealed non-necrotizing granulomata, consistent with sarcoidosis. Acid-fast and Grocott's methenamine silver stainswere negative for mycobacteria and fungi, respectively. Cultures for these organisms remained negative. Pulmonary and cardiomyopathy c5onsultations were obtained; corticosteroid therapy was not recommended. Treatment of LV dysfunction with beta-blockers and angiotensin converting enzyme (ACE) inhibitors was instituted.

An echocardiogram performed 4 months after ICD placement showed mild global LV hypokinesis, with LVEF $40-45 \%$. This remained essentially the same one year after the device implantation (LVEF 40-45\%). The device interrogation revealed predominant atrial sensed-ventricular paced (As-Vp) pattern with DDD 50-130 programming, and occa- 

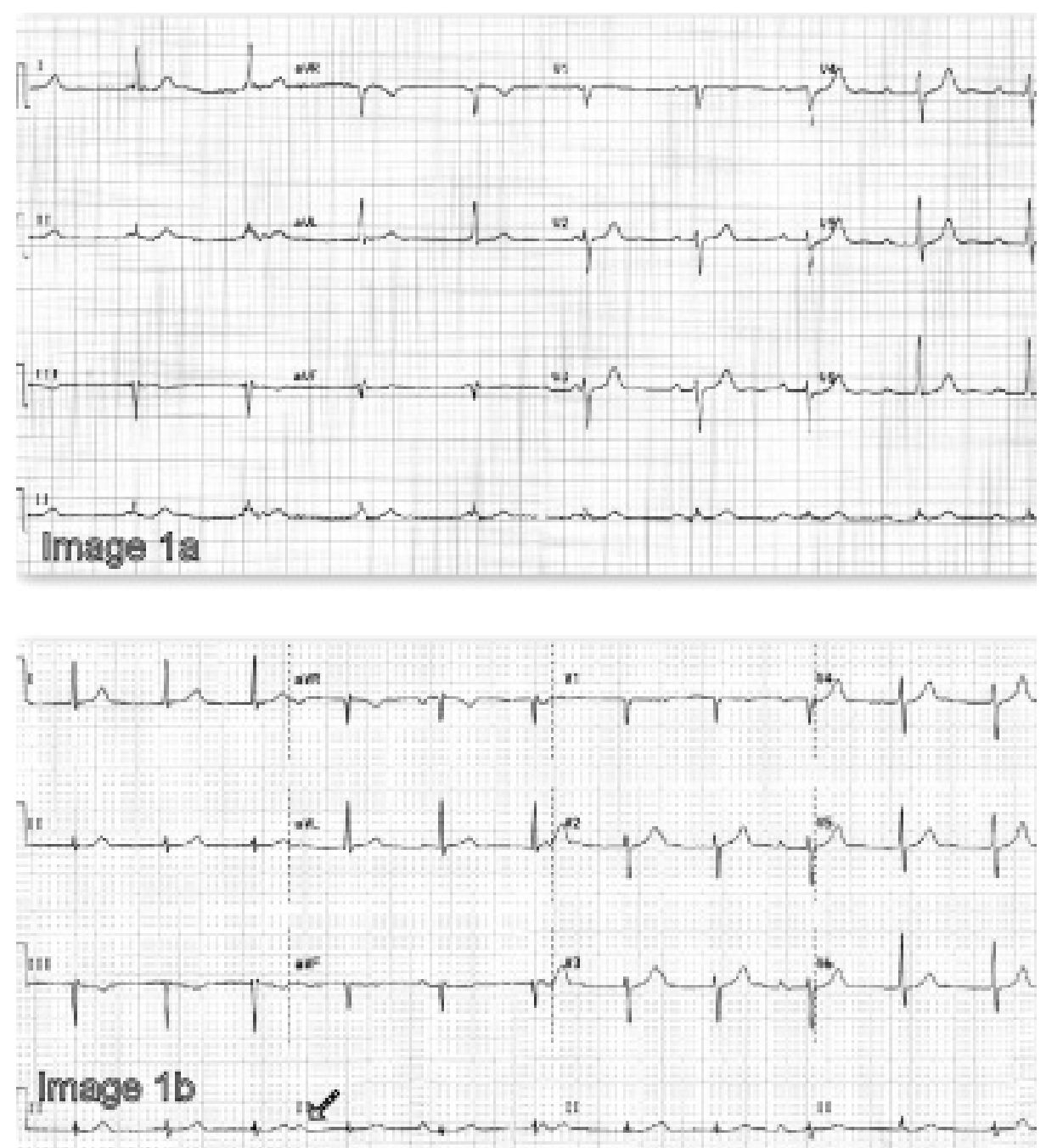

Fig. (1). Two examples of 12-lead ECG prior to device implantation. a Sinus rhythm (60-64 bpm) with complete heart block and slightly slower junctional escape rhythm $(58 \mathrm{bpm})$. b Sinus bradycardia $(53 \mathrm{bpm})$ and accelerated junctional rhythm $(68 \mathrm{bpm})$, resulting in AV dissociation. Arrow indicates a sinus P wave which should have been conducted if AV block had not been present. Instead, the long PR interval and constant RR intervals indicate a combination of AV block and accelerated junctional rhythm.

sional episodes of junctional tachycardia in the $140-160 \mathrm{bpm}$ monitoring-only slow Ventricular tachycardia zone. His symptoms of mild exercise intolerance remained unchanged.

\section{DISCUSSION:}

Sarcoidosis is a systemic infiltrative, non-caseating granulomatous disease of unknown etiology [1, 2]. Cardiac involvement can clinically manifest as cardiac arrhythmia or heart failure. AV block, presumably due to AV nodal involvement with the disease, is the most frequent presentation, although ventricular tachycardia due to reentry around a ventricular scar resulting from healing of the active disease process is not uncommon and can result in sudden cardiac death (SCD). If extensive, scaring can cause severe LV dysfunction and heart failure. Diagnosis of cardiac sarcoidosis can be difficult, especially if extracardiac involvement is absent or not apparent [3]. The guidelines for diagnosis of cardiac sarcoidosis proposed by Japanese Ministry of Health and Welfare may underestimate its true prevalence [4].

The risk-stratification with respect to SCD is difficult because of relative rarity of this condition; no randomized studies comparing different strategies are available. Most electrophysiologists would recommend ICD placement in patients with sustained ventricular arrhythmias and in those with severe LV dysfunction, employing analogy with myocardial scarring due to prior myocardial infarction $[2,5]$. The management of the other patients with respect to SCD protection is more controversial. It appears that programmed ventricular stimulation (PVS) does have a prognostic value, although even patients with negative PVS carried a risk of $\operatorname{SCD}(2 / 22$ patients over 34 months follow-up) in a mediumsized series [6].

In this patient, the decision for ICD implantation was driven by the established need for a cardiac-rhythm management device and uncertainty about the magnitude of risk of death due to ventricular tachyarrhythmia, which could be on the order of a few percent a year even if the PVS had been negative. Interestingly, relatively frequent episodes of accelerated junctional rhythm persisted for months; to the best of our knowledge, this has not been reported before in cardiac sarcoidosis, though it is not completely unexpected if AV node is involved by the granulomatous process. 

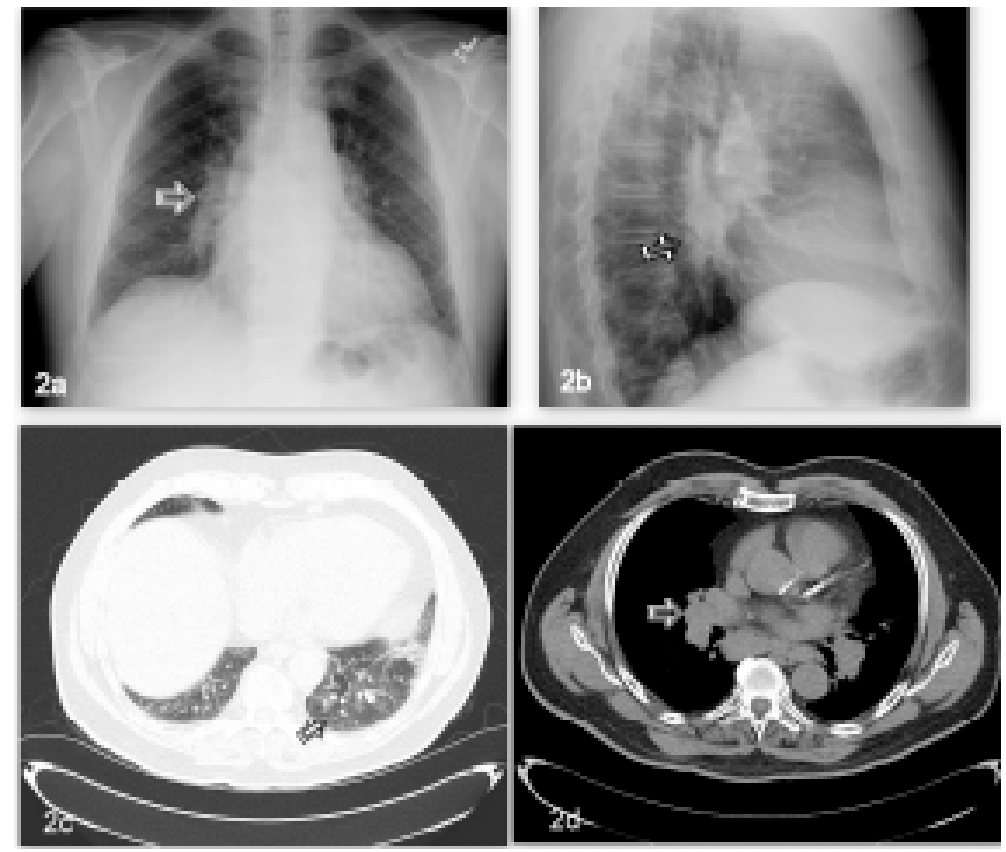

Fig. (2). a, b PA and lateral CXR, suggesting hilar enlargement (arrows). c, d CT of the chest without contrast, confirming hilar lymphadenopathy (d) and interstitial lung disease with scarring and nodules (c). The CT findings are consistent with stage 2 pulmonary sarcoidosis

Treatment with systemic corticosteroids is effective for pulmonary sarcoidosis and is often used in patients with cardiac involvement, although its efficacy is only supported by case reports rather than randomized trials [3]. We considered systemic steroids in this patient, but given the mild symptoms and potential for serious side-effects of steroid therapy, this was not advised by either pulmonary or cardiomyopathy specialists. This patient's cardiac status now appears stable on medical treatment of his mild LV dysfunction. Annual follow-up of LV function with echocardiography is planned.

\section{REFERENCES}

[1] Nunes H, Brillet PY, Valeyre D, Brauner MW, Wells AU. Imaging in sarcoidosis. Semin Respir Crit CareMed 2007; 28: 102-20.
[2] Soejima K, Yada H. The work-up and management of patients with apparent or subclinical cardiac sarcoidosis: with emphasis on the associated heart rhythm abnormalities. J Cardiovasc Electrophysiol 2009; 20: 578-83.

[3] Doughan AR, Williams BR. Cardiac sarcoidosis. Heart 2006; 92: 282-8.

[4] Patel MR, Cawley PJ, Heitner JF, et al. Detection of myocardial damage in patients with sarcoidosis. Circulation 2009; 120: 196977.

[5] Myerburg RJ, Reddy V, Castellanos A. Indications for implantable cardioverter-defibrillators based on evidence and judgment. J Am Coll Cardiol 2009; 54: 747-63.

[6] Aizer A, Stern EH, Gomes JA, Teirstein AS, Eckart RE, Mehta D. Usefulness of programmed ventricular stimulation in predicting future arrhythmic events in patients with cardiac sarcoidosis. Am J Cardiol 2005; 96: 276-82. 